\title{
Development of an ANFIS Neural Network System for Early Diagnosis of Critical Health Condition of a Patient
}

\author{
Taksala Devapriya A. ${ }^{1}$, Deepesh B. ${ }^{2}$ \\ ${ }^{I}$ (Electronics and Communication Engineering, Mountzion College of Engineering and Technology/Anna \\ University, India) \\ ${ }^{2}$ (Electronics and Communication Engineering, Mountzion College of Engineering and Technology/Anna \\ University, India)
}

\begin{abstract}
Aim of this work is to design and training of a ANFIS neural network based early diagnosis of a patient through a smart instrument. The data collected from the patient is used to train the Neural Network. The rules are designed on the basis of the collected data. The data collected from the patients are weight, BMI, Glucose, Creatinine, Systolic BP, and Diastolic BP. The rules for system are given according to the characteristics of the data obtained from patients. The output is mainly classified into three classes that are severe, moderately critical, and normal. Output of Neural Network is connected to LED to display the corresponding outputs. The advantage of this system is we can implement this device as portable. Hence we can easily monitor the patient's condition at anywhere. And for the normal type of application we need a personal computer to compute the inputs and outputs. Because here we are using FPGA the problem can be easily avoided and also PC will need an uninterrupted power supply. For this device just battery power is enough to work. The FPGA will take a very small power only hence we can easily use the device a long time without charging again and again. These are some of the major advantages of Smart device. The main disadvantage of using fuzzy logic is the reconfiguration problem. When we change the input parameters we should also change the rules. That is one of the important disadvantages of the fuzzy logic. This will also affect the cascaded structure of the neural network. Hence the performance of the system will effect sufficiently. The changing of rules can do only by the technical experts. This also will increase the complexity of the system. The problem can be easily solved by using ANFIS instead of fuzzy controllers. By using this we can avoid the re configuration complexity problem. Because ANFIS generates rules automatically from the training data. Hence no need for giving rules externally. Hence we can change the input data as per the user wish. Training complexity can be reduced by this method.
\end{abstract}

Keywords: Fuzzy Logic, ANFIS, ANN, SSA

\section{INTRODUCTION}

TIn the Modern world peoples are living with different environmental condition has different physical problems because of Natural, psychological and personal duels. Medical diagnosis and decision making is a complicated and judgmental process. Diagnostic decisions made by physicians are highly variable ( It may change from one physician to other ). It is not based only on medical knowledge derived from books and literatures and data obtained from various pathological tests, but also depend largely on experience, judgment and reasoning which essentially are the functions of human brain. However, in third world countries like India, doctors are scarcely available in rural areas. A recent statistical data shows that $75 \%$ of qualified consulting doctors reside in urban areas and another $23 \%$ in semi-urban areas, and only about $2 \%$ of doctors reside in rural areas, where, unfortunately, nearly $78 \%$ of Indians reside. This has created an unwarranted imbalance in patient-doctor ratio of more than 10,000 patients for one doctor in rural India. Apart from the acute scarcity of physicians, lack of availability of electricity is quite common in rural areas of third world countries like India. Such prosaic problems necessitate the use of an inexpensive, portable, low power battery operated high-speed. Recent development in the communication system is one of the big reliefs in medical side because of faster data transmission at the severe condition of a patient. It is helping to make the decision by a group of physician in different places. It is increasing the accuracy of the decision and the physicians can take turn the correct direction for the better diagnosis. Use of telecommunication to transmit health-related data to a remote centre for necessary help, has been the issue over the last several decades. Starting with a simple telephonic conversation with a doctor, the advent of internet technologies revolutionized the scenario. Using e-mail for sending patient data, sending images and physiological signals over internet by store and forward method and final videoconferencing all improved drastically tele-medicine setup for referral health services mainly. But everywhere, data, image and signals were transmitted for the ultimate decision taking by human brains. In the third world countries, an instrument with some auto decision making support can be used as a preventive device for early diagnosis of problems related to specified systems of patients' bodies. Especially in rural areas, where doctors 
are scarcely available. Moreover, each doctor in these countries have to handle large number of patients, it may become handy for a doctor to keep track of the previous data of a particular patient when preservation of data and documents, like investigation reports, is poor in rural backdrop. This research focuses on the development of smart processing system that can predict the physiological state of a patient, given the past physiological data of the patient. The scheme under consideration can provide alarm to the relevant personnel in advance before the patient reaches a critical state, who would then contact the physician at the remote site. It will be very helpful to give a good diagnostic to the patient by proper medicine or surgery. The smart processing system consists of blocks for ANFIS, NNT and display unit. It can serve to handle the peripheral health screening of patients and thus screen out the few critical patients out of many who really need clinical assistance from the physician. In order to save the number of combinational logic blocks in implementing the system, the division process required for normalization of membership functions is implemented using intelligent multiplication techniques. Among other benefits are the short design cycle and the scope of re-programmability for improvement in the design without any additional cost along with the facility of desktop testing. The system can be used for diagnosis of other disease like diabetes and heart related problems.

\section{FUNCTIONAL ARCHITECTURE OF SMART AGENT}

\subsection{The Smart Agent}

Earlier diagnosis of a patient can be work out by using a smart agent. It is the combination of artificial intelligence algorithms such as ANFIS (Adaptive Neuro Fuzzy Inference System) and ANN (Artificial Neural Network). The schematic block diagram of the proposed architecture can be shown in Fig. 1. It has the cascaded structure of ANFIS and Neural Network.The patients sample data is used to train the Neural Network and ANFIS. The attributes taken from the patients are given below. These six Attributes are entirely correlated with the renal failure. They are given below.

1. B.M.I (Body Mass Index)

2. Blood Glucose

3. Creatinine

4. Urea

5. Systolic Blood Pressure

6. Diastolic Blood Pressure

The data from the patient such as height or weight data cannot always be trusted as they are subjected to the quality and accuracy of measuring units and the skill of the technician.

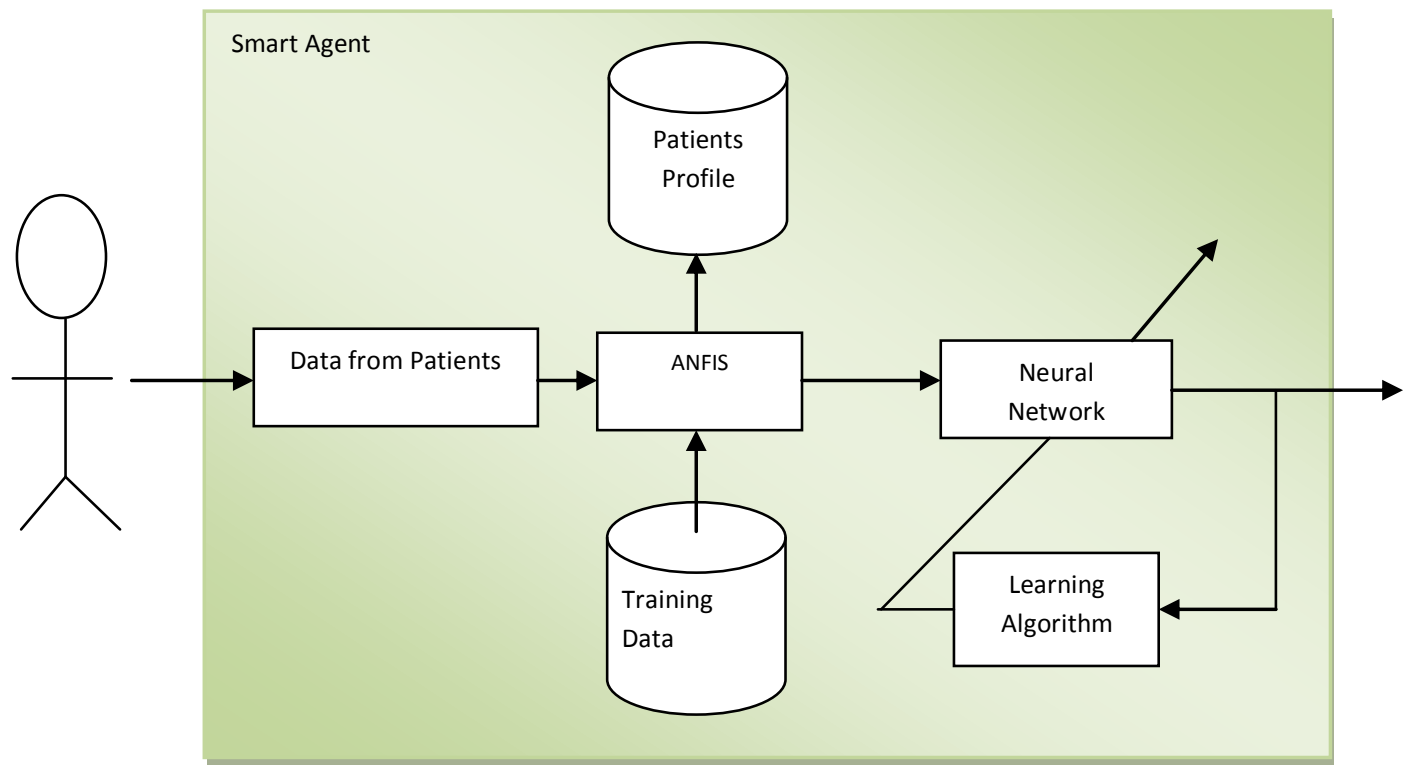

Fig.2. Block Diagram of Proposed Method

Moreover, based on a single data, it would be highly uncertain to make an accurate decision about the future physiological state of the patient. So the patient data have been fuzzified with the objective of transformation of periodic measures into likelihoods that the body mass index, blood glucose, urea, creatinine, systolic and 
diastolic blood pressure of the patient is high, low or moderate. It has been established for fuzzification of patient data. These attributes are fully correlated with the renal failure. Hence the degree of accuracy is also depends upon the selected attributes.

\subsection{ANFIS Training}

ANFIS is trained with the patient's data samples with six attributes. Target for ANFIS is given by three classes. Three classes used to predict the system are:

\section{Normal}

2. Critical

3. Severe

The Dataset used to train is National renal database. In this the information collected is by 40 days interval. These data is collected and it is pre-processed i.e. aligned in a The data from the patient such as height or weight data cannot always be trusted as they are subjected to the quality and accuracy of measuring units and the skill of the technician. Moreover, based on a single data, it would be highly uncertain to make an accurate decision about the future physiological state of the patient. So the patient data have been fuzzified with the objective of transformation of periodic measures into likelihoods that the body mass index, blood glucose, urea, creatinine, systolic and diastolic blood pressure of the patient is high, low or moderate ${ }^{[1]}$. It has been established for fuzzification of patient data. These attributes are fully correlated with the renal failure. Hence the degree of accuracy is also depends upon the selected attributes.

\subsection{Neural Network Training}

Figure 2 shows the structure of neural network used. The left most layer is the input layer, which accepts the membership function values, which are presented to the hidden layer. The input layer consists of 60 neurons, the hidden layer consists of 18 neurons and the output layer consists of three neurons.

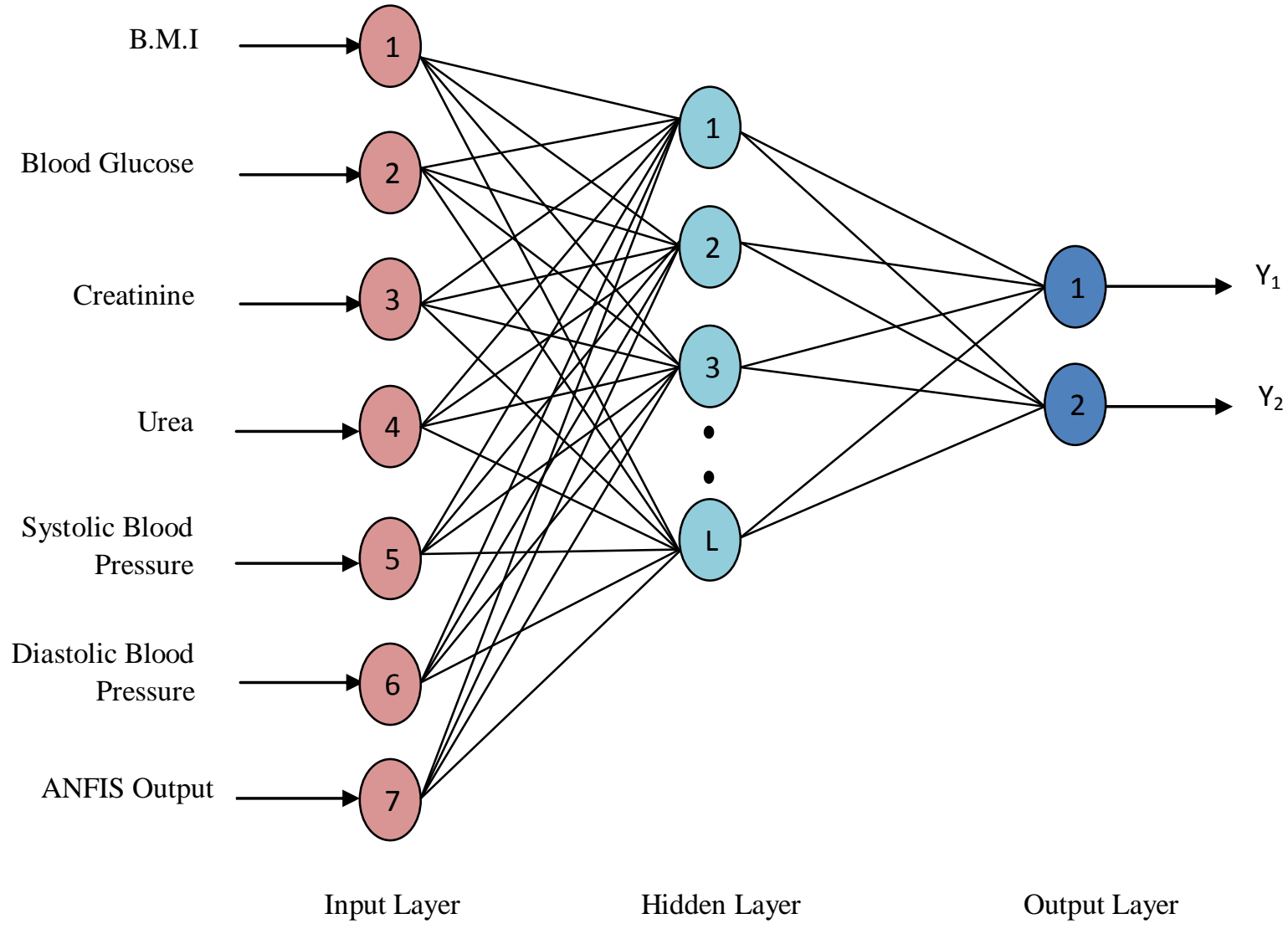

Fig.2. Neural Network Structure

The neural network calculates the possibilities of the pathophysiological parameters to below, moderate or high at the next instant of time ${ }^{[2]}$. The connection between the input layer and the hidden layer is shown as interconnection network box for clarity. There is a connection between every neuron in the input layer and every neuron in the hidden layer. The weighted sum of the possibility values from the hidden to the output layer 
indicates the criticality of the current condition of the patient which is indicated as an output of the neural network.

\subsection{Mathematical model of Neurons}

\section{INDENTATIONS AND EQUATIONS}

The neural network proposed in the current work is based on inverse delayed function model of neuron ${ }^{[3]}$. The inverse delayed function model of neuron proposed is given by the following set of differential equations:

$\tau \frac{d u}{d t}=\sum w_{i j} x j+a_{i i} x_{i}-u_{i}$

$\tau_{x} \frac{d x_{i}}{d t}=u_{i}-g\left(x_{i}\right)$

$g\left(x_{i}\right)=f^{-1}\left(x_{i}\right)-K x_{i}$

Where $\mathrm{w}_{\mathrm{ij}}$ is the synaptic weight between $\mathrm{i}^{\text {th }}$ and $\mathrm{j}^{\text {th }}$ neurons, $\mathrm{a}_{\mathrm{ii}}$ is the synaptic weight of self-connection, $\mathrm{u}_{\mathrm{i}}$ is the internal state of $\mathrm{i}^{\text {th }}$ neuron, $\mathrm{x}_{\mathrm{j}}$ is the output of the $\mathrm{j}^{\text {th }}$ neuron, $\boldsymbol{\tau}$ and $\boldsymbol{\tau}_{\boldsymbol{x}}$ are the time constant of the internal state and time constant of the neuron output, respectively. If $f(x)$ is the sigmoid function used in a conventional neural network, and then $\boldsymbol{g}\left(\boldsymbol{x}_{\boldsymbol{i}}\right)=\boldsymbol{f}^{-1}\left(\boldsymbol{x}_{\boldsymbol{i}}\right)$ is the N-shaped inverse output function and $g(x)$ can be changed with the positive parameter $\mathrm{K}$.

The conversion from $\mathrm{u}$ to $\mathrm{x}$ has a very small response time compared to $\tau$ and thus $\tau_{x} \ll \tau_{x}$. However, the conversion time should take into consideration in general cases under the condition that it is much smaller than $\tau_{x}$.

$\tau_{x^{\prime}} \frac{d^{2} x_{i}^{i}}{d t^{2}}=\frac{d u_{i}^{n}}{d t}-\frac{d g\left(x_{i}\right)}{d x_{i}^{i}} \cdot \frac{d x_{i}^{i}}{d t}$

Let

$\phi_{i}=\frac{d g\left(x_{i}\right)}{d x_{i}} \cdot \frac{\tau_{\underline{x}}}{\tau}$

Putting Eq. (6) in (7) we get

$\tau_{x} \frac{d^{2} x_{i}}{d t^{2}}+\phi_{i} \frac{d x_{i}}{d t}-\frac{\tau_{x}}{\tau} \cdot \frac{d x_{i}}{d t}=\frac{d u_{i}}{d t}$

$\Leftrightarrow \tau_{x} \frac{d^{2} x_{i}}{d t^{2}}+\phi_{i} \frac{d x_{i}}{d t}=\frac{1}{\tau}\left(\sum_{j} w_{i j} x_{j}-g\left(x_{i}\right)\right)$

$\frac{\partial U_{i}}{\partial x_{i}}=\frac{1}{\tau}\left(g\left(x_{i}\right)-\sum_{j} w_{i j} x_{j}\right)$

Therefore, Eq. (8) can be expressed as

$\tau_{x} \frac{d^{2} X_{i}}{d t^{2}}+\phi_{i} \frac{d x_{i}}{d t}=-\frac{\partial v_{i}^{i}}{\partial x_{i}}$

Where

$$
U_{i}=\frac{1}{\tau}\left(\int_{0}^{x_{i}} g\left(X_{i}\right) d x_{i}-X_{i} \Sigma_{j} w_{i j} X_{j}\right)
$$

$U_{i}$ represents the potential of the inverse delayed function model. Eq. (9) corresponds to the kinetics of a particle in presence of frictional forces. The first term in Eq. (9) represents inertia and the second term represents friction. If $\mathrm{g}$

$\left(\mathrm{x}_{\mathrm{i}}\right)$ is an $\mathrm{N}$ shaped function and $\frac{d g\left(x_{\bar{i}}\right)}{d x_{\bar{i}}}$ is $<-\tau_{x} / \tau$ over a certain range of $\mathrm{x}_{\mathrm{i}}$, (since $\tau_{x} / \tau$ is always positive) then the inverse delayed model has the effect of negative resistance $\left(\phi_{i}<0\right)$. The energy function of the inverse delayed function model using Lyapnov function is given by

$E=-\frac{1}{2 \pi} \sum_{i} \sum_{j} w_{i j} x_{i} x_{j}+\frac{1}{\tau} \sum_{i} \int_{0}^{x_{i}} g\left(x_{i}\right) d x_{i}+\frac{\pi_{X}}{2} \sum_{i}\left(\frac{d x_{i}}{d t}\right)^{2}$

Where the self-connection between the neurons is ignored, since there is no self-connection between the neurons in our network. The last term appears in Eq.(11) because of time delay introduced in the inverse delayed function model. Differentiating both sides of Eg. (11) with respect to time t, we get

$\frac{d E}{d t}=-\sum_{i} \frac{d x_{i}}{d t}\left\{\frac{1}{\tau} \sum_{j} w_{i j} x_{j}-\frac{1}{\tau} g\left(X_{i}\right)-\tau_{x} \frac{d^{2} x_{i}}{d t^{2}}\right\}$ 
$\Leftrightarrow \frac{d E}{d t}=-\sum_{i}\left(\frac{d g\left(x_{i}\right)}{d x_{i}}+\frac{1}{\tau}\right)\left(\frac{d x_{i}}{d t}\right)^{2}$

$\Leftrightarrow-\Sigma_{i} \phi_{i}\left(\frac{d x_{i}^{i}}{d t}\right)^{2}$

From Eq.(14), we find that the time evolution of the system is a motion in the states pace that seeks out minima in $\mathrm{E}$ and comes to a stop at all such points if $\phi_{i}$ is positive. However, if $\phi_{i}$ is negative, the energy function does not monotonically decrease and can climb up the potential hill because of the effect to $\mathrm{f}$ negative resistance and can destabilizes table equilibrium points of the network within the negative resistance area. This can be made possible by harnessing the decreasing slope of $\mathrm{N}$ shaped characteristic of inverse a sigmoid activation function ${ }^{[4]}$. Hence, a neural network to answer an optimization problem can be constructed, as all units in the network should be at the corners of the solution space when its motion comes to as to pate global minima. On the other hand, when it comes to stop at the local minima, some of the units in the network are in the inside of the solution space. Therefore, it is expected to increase the possibility to get out from the local minima, if the network is an inverse delayed function model.

Neural Network Having 7 inputs because it is a cascaded structure of ANFIS and neural Network. One input is coming from the ANFIS and other six inputs are the six attributes. Hence the neural networks efficiency is doubled.

\section{FIGURES AND TABLES}

As discussed above the ANFIS have six inputs. These six inputs are the six important attributes which are correlated with the renal diseases. Degree of prediction accuracy can be increased by increasing the number of dependent attributes. Hence the attributes should be selected in a proper manner that the increasing orders of priority. Sample patients information is given below. The following table data is used to train the ANFIS and Neural Network. The Neural Networks Accuracy is depending upon the number of Neurons in the hidden layer. The accuracy of the system is directly proposed to the Number of neurons. Even though, the system complexity will be increase if the number of neurons increases. Because the arithmetic and logical operations will increases if the number of neurons increases. Hence we should select the optimum number of neurons. It should depend upon the accuracy and the complexity of the developmental system.

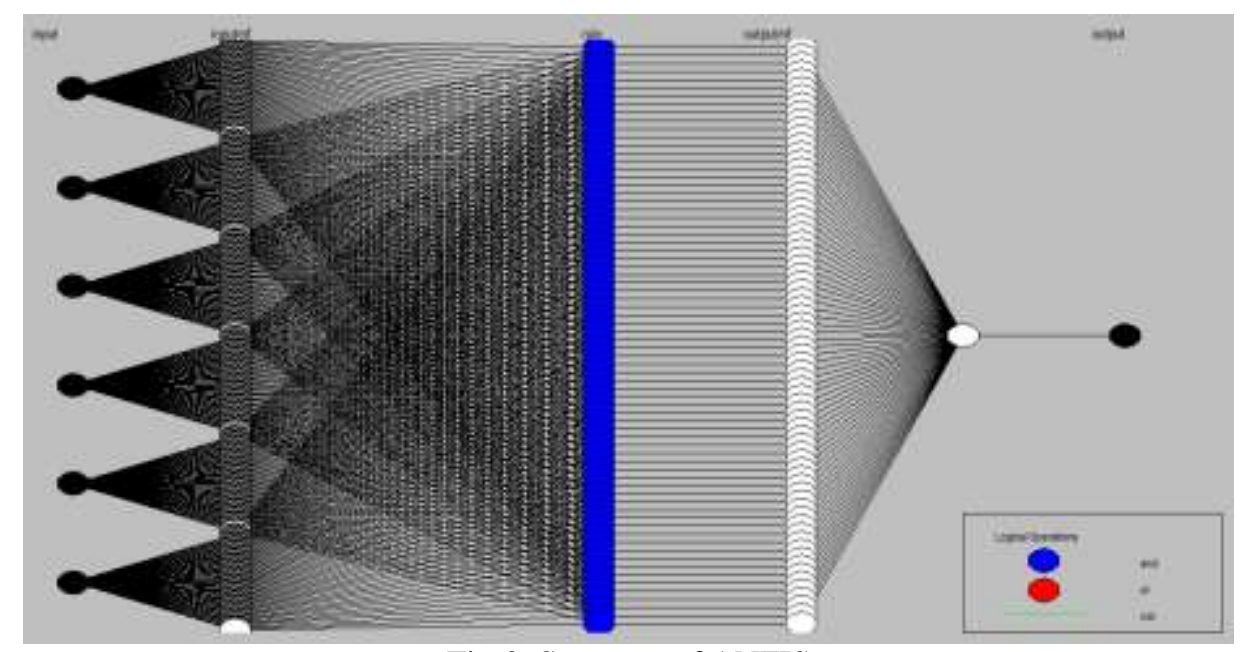

Fig.3. Structure of ANFIS

Similarly for ANFIS the training is performed on the basis of subtractive clustering algorithm. The training of ANFIS is done by ten epochs. The minimum gradient error will reach within this epoch is considering as the target. The training duration is depending upon the size of the dataset. If the size is very high training will take more time and also the ANFIS will generate a complex structure. Hence it is ensure that the number of epochs and the size of the training data should be select in an optimized way. It will increase the accuracy and reduce the complexity of the system. At the implementation view the complexity of the system should be low and also the accuracy should be very high.

The following surface plots show the membership function for the different attributes. Y-axis shows the degree of membership and $\mathrm{X}$-axis and $\mathrm{Z}$-axis are the corresponding attributes. The $\mathrm{Y}$-axis is varies from 0 to 1.0 represents the lower priority and 1 represent varies to the higher priority. The membership function varies in a linear fashion. The value 0 to 1 represents the Normalized value. For hardware implementation we can consider this value as 8 bit or 16 bit according to the user requirement. Normally it is the type of the processor or DSP 
used. Increased number of bits will increase the resolution of the system. For the FPGA implementation we should select the number of bits according to the device used. Normally half precision floating point number is used. It has a maximum value of +1 and a minimum value of -1 . Normally 16-bit is used to represent half precision fixed point number. 1 bit is used to represent the sign and remaining bits are used to represent the floating point representation

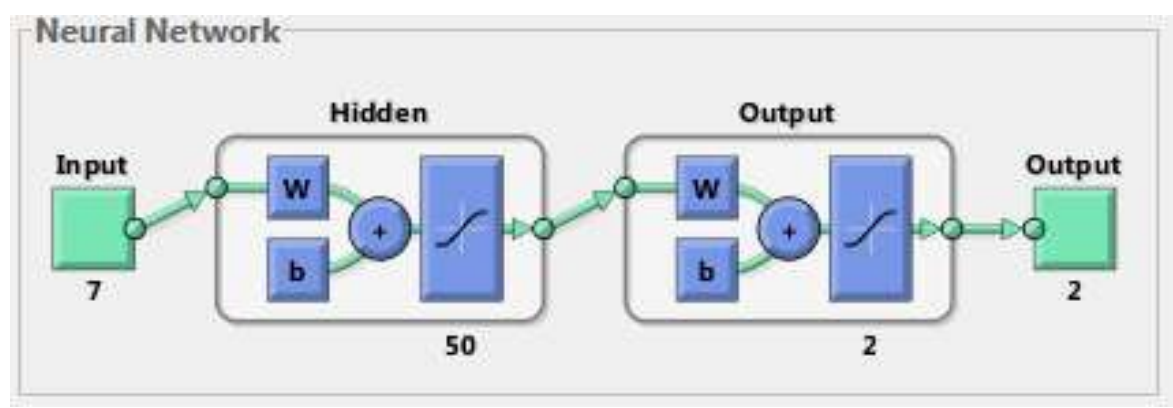

Fig.4. Structure of Neural Network

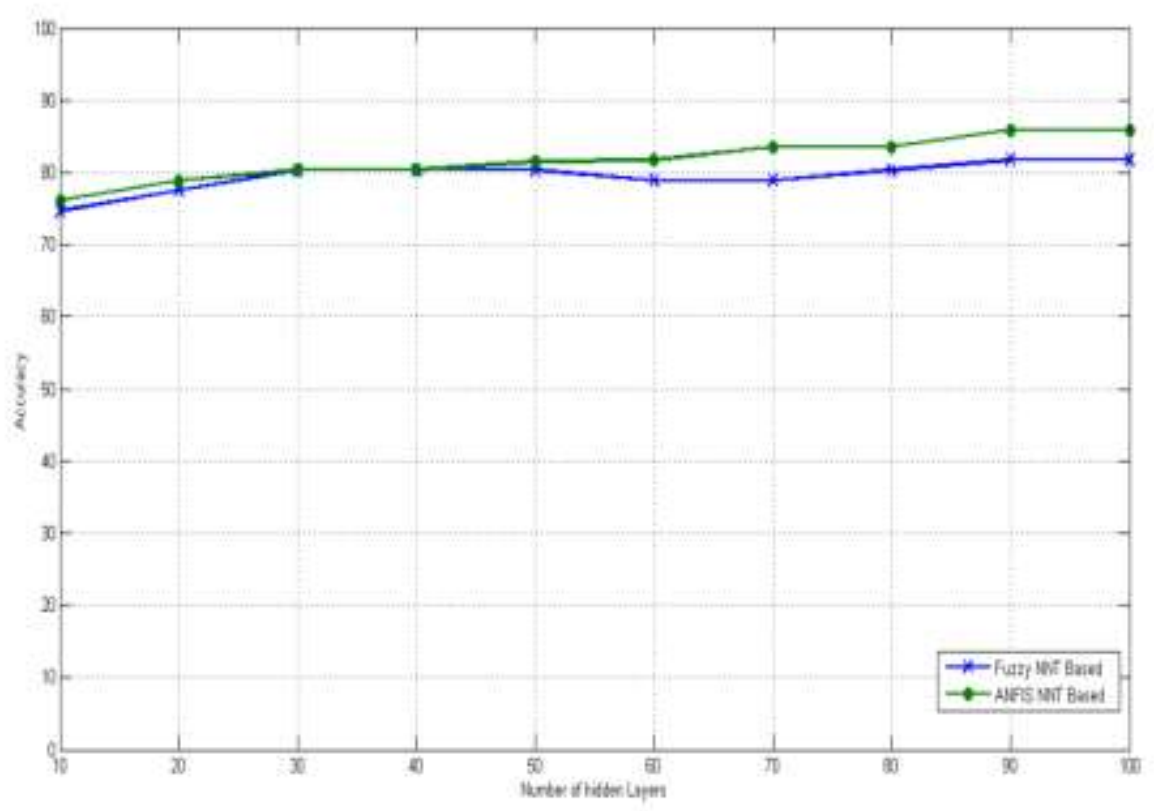

Fig.5. Performance

\begin{tabular}{|l|l|l|l|l|l|l|l|l|l|l|}
\hline $\begin{array}{l}\text { No of } \\
\text { Neurons }\end{array}$ & 10 & 20 & 30 & 40 & 50 & 60 & 70 & 80 & 90 & 100 \\
\hline $\begin{array}{l}\text { ANFIS } \\
\text { \&NNT }\end{array}$ & 76.1 & 78.9 & 80.3 & 80.3 & 81.5 & 81.7 & 83.5 & 83.5 & 85.9 & 85.9 \\
\hline $\begin{array}{l}\text { Fuzzy } \\
\text { \&NNT }\end{array}$ & 74.6 & 77.5 & 80.3 & 80.3 & 80.3 & 78.9 & 78.9 & 80.3 & 81.7 & 81.7 \\
\hline
\end{tabular}

Table.1. Performance

\section{Conclusion}

The current work focuses on the application of Neuro-fuzzy synergism to detect at an early stage the probable approaching critical condition of a patient. It explains the usage of ANFIS neural networks in medical diagnosis systems and the extended example on the problem of early detection of approaching critical renal condition of patients. In order to improve the accuracy of diagnosis, optimal synaptic weights have been found out using the inverse delayed function model of the neuron. The neurons that are considered in the proposed network are devoid of self connections unlike the previously proposed networks based on inverse delayed function model of neurons where self-connected neurons have been assumed. For diagnosis purposes, body mass index (B.M.I.), glucose, urea, creatinine, systolic and diastolic blood pressures are considered as 
pathophysiological parameters. The ANFIS neural network has been suitably trained and tested with real patient data to find out the correspondence with the decision being given by the fuzzy neural network and the actual pathophysiological state of the patient. A reasonably high accuracy of $85 \%$ in medical diagnostic decisionmaking has been obtained with the implemented system.

\section{Journal Papers:}

\section{REFERENCES}

[1] M Venkatesan, Classification of Renal Failure Using Simplified Fuzzy Adaptive Resonance Theory Map, IJCSNS International Journal of Computer Science and Network Security, VOL.9 No.11, November 2009.

[2] D. Sheppard,Predicting cytomegalovirus disease after renal transplantation: an artificial neural network approach,International Journal of Medical Informatics 54 (1999) 55-76.

[3] ZaritaZainuddin, A Neural Network Approach in Predicting the Blood Glucose Level for Diabetic Patients, International Journal of Information and Mathematical Sciences 5:1 2009.

[4] Adenike O. Osofisan, Prediction of Kidney Failure Using Artificial Neural Networks, European Journal of Scientific Research ISSN 1450-216X Vol.61 No.4 (2011), pp.487-492

\section{Author's Profile}

Fdsfgsgdfgdfg 